\title{
Integration von Genesungsbegleitern in psychiatrische Behandlungsteams
}

\author{
Studiendesign, Baseline-Daten und erste Ergebnisse \\ einer qualitativ-empirischen Interviewstudie
}

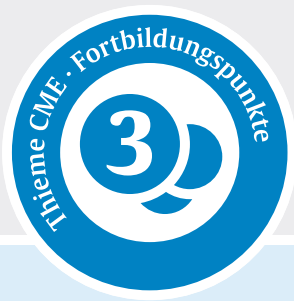

Jakov Gather1,2*, Ina Otte ${ }^{2 *}$, Anna Werning1, Alexa Nossek ${ }^{2}$, Jochen Vollmann ${ }^{2}$, Georg Juckel ${ }^{1}$

1 LWL-Forschungsinstitut für Seelische Gesundheit, LWL-Universitätsklinikum, Ruhr-Universität Bochum

2 Institut für Medizinische Ethik und Geschichte der Medizin, Ruhr-Universität Bochum

\section{ZUSAMMENFASSUNG}

Gegenstand und Ziel Wie können ausgebildete Genesungsbegleiter (GB) in psychiatrische Behandlungsteams in Akutkliniken integriert werden? Welche Rollen und Aufgaben übernehmen sie? Welche Hindernisse treten auf und welche Strategien werden zu ihrer Überwindung entwickelt? Welche spezifischen Fähigkeiten besitzen GB? Welchen Einfluss können sie auf die Anwendung von Zwangsmaßnahmen haben?

* geteilte Erstautorenschaft
Material und Methoden Baseline-Erhebung mittels Kurzfragebögen in 5 psychiatrischen Kliniken des Landschaftsverbands Westfalen-Lippe. Anschließend qualitativ-empirisches Studiendesign und Auswertung der Daten mittels qualitativer Inhaltsanalyse nach Mayring.

Ergebnisse GB nehmen eine Vielzahl an unterschiedlichen Aufgaben wahr und können im Akutbereich dazu beitragen, Zwangsmaßnahmen vorzubeugen.

Schlussfolgerungen Die Unterstützung durch Ansprechpartner in den Kliniken sowie überregionale Treffen inklusive Supervisionsangebot sind für die Integration hilfreich, da sie Austausch zwischen den GB ermöglichen und Aufgaben- und Rollenfindung erleichtern.

Klinische Relevanz Das Projekt zeigt, wie GB in psychiatrische Behandlungsteams in Akutkliniken integriert werden können und welchen spezifischen Beitrag, u.a. im Hinblick auf eine Reduktion von Zwangsmaßnahmen, sie leisten können.

\section{Einleitung}

Die Idee, von einer psychischen Erkrankung betroffene Menschen im Sinne eines „Experienced-Involvement (EXIN) “ als Genesungsbegleiter an der medizinischen Behandlung anderer Menschen zu beteiligen, hat im Hinblick auf eine Implementierung im psychiatrischen Versorgungssystem und eine wissenschaftliche Evaluation erst in den letzten beiden Jahrzehnten eine nennenswerte Ausweitung erfahren [1]. Während in angelsächsischen Ländern die „Peer-Arbeit“ als Teil des psychiatrischen Behandlungsangebotes vielerorts anerkannt und etabliert ist [2], finden sich vergleichbare Ansätze in Deutschland bislang noch selten. Seit einigen Jahren gibt es für Deutschland ein einjähriges Ausbildungscurriculum zum Genesungsbegleiter, das in einem europäischen Projekt entwickelt wurde und mittlerweile an zahlreichen Schulungsorten angeboten wird [3, 4]. Dies hat zur Folge, dass auch in Deutschland immer mehr Genesungsbegleiter nach einer Möglichkeit suchen, eine Anstellung im psychiatrischen Versorgungssystem zu erhalten und ihr spezifisches Wissen sowie ihre eigenen Erfahrungen mit psychischen Krisen in die Behandlung psychisch kranker Menschen einzubringen. Der Landschaftsausschuss des Landschaftsverbands Westfalen-Lippe (LWL) initiierte im Jahr 2011 einen Aktionsplan zur Umsetzung der UN-Behindertenrechtskonvention, der im Handlungsfeld „Gesundheit“ das Projekt „Einsatz und Bezahlung von ausgebildeten Genesungsbegleitern im LWL-PsychiatrieVerbund Westfalen" enthielt (Laufzeit 2015-2017). Dieses Projekt beinhaltete eine zweijährige finanzielle Förderung der Anstellung von ausgebildeten Genesungsbegleitern und hatte zum Ziel, den Einsatz von Genesungsbegleitern innerhalb des LWL-PsychiatrieVerbunds voranzutreiben. Entwickelt wurde das Projekt aufseiten des LWL durch den multiprofessionell und trialogisch besetzten Arbeitskreis (AK) „Trialog konkret“, der das Projekt während der Laufzeit kontinuierlich koordinierte. Im Mai 2015 wurde allen Einrichtungen im LWL-PsychiatrieVerbund die Möglichkeit gegeben, sich für die Teilnahme an dem Projekt zu bewerben und Genesungsbegleiter einzustellen. Schlussendlich wurden in den LWL-Kliniken Gütersloh, Herten, Lengerich, Marsberg und Münster insgesamt 6 Genesungsbegleiterinnen und 2 Genesungsbegleiter (im Folgenden alle 8 als Genesungsbegleiter bezeichnet) in die Evaluation aufgenommen.

Mit der wissenschaftlichen Begleitung des Projekts wurde das LWL-Forschungsinstitut für Seelische Gesundheit am LWL-Universitätsklinikum der Ruhr-Universität Bochum (RUB) beauftragt, das hierfür über die gesamte Projektlaufzeit mit dem Institut für Medizinische Ethik und Geschichte der Medizin der RUB kooperiert hat. Die Ethik-Kommission der Medizinischen Fakultät der RUB hat die hier vorgestellte Studie beraten und zustimmend bewertet (Registrier-Nr.: 15-5387). 
- Tab. 1 Studiendesign

\begin{tabular}{|c|c|c|c|}
\hline Studienphase & Methoden & Studienteilnehmer & Zielsetzung \\
\hline \multirow{3}{*}{$\begin{array}{l}\text { Initiale Implemen- } \\
\text { tierungsphase / } \\
\text { Baseline-Daten- } \\
\text { erhebung }\end{array}$} & a) Sitzung zur Aufgabendefinition der GB & a) GB und PDL & $\begin{array}{l}\text { a) Austausch über Vorerfahrungen, Diskus- } \\
\text { sion von Aufgabengebieten }\end{array}$ \\
\hline & b) Vortrag durch Projektmitarbeiter & $\begin{array}{l}\text { b) GB, PDL sowie gesamtes Team der } \\
\text { Station }\end{array}$ & $\begin{array}{l}\text { b) Vermittlung der Hintergründe zu EX-IN, } \\
\text { Vorstellung des Forschungsprojekts }\end{array}$ \\
\hline & c) standardisierte Befragung & c) GB und PDL & $\begin{array}{l}\text { c) Erhebung von Eckdaten zur Arbeit und } \\
\text { Bezahlung von } \mathrm{GB}\end{array}$ \\
\hline \multirow{3}{*}{$\begin{array}{l}\text { Feldkontakt 1: } \\
\text { „Beobachtungen“ }\end{array}$} & offene, nicht teilnehmende Beobachtung von & & \multirow{3}{*}{$\begin{array}{l}\text { Ermittlung der Rollen der GB innerhalb } \\
\text { von teaminternen Kommunikations- und } \\
\text { Entscheidungsprozessen, Potentiale und } \\
\text { Hindernisse bei der Integration der GB in } \\
\text { Behandlungsteams }\end{array}$} \\
\hline & a) multiprofessionellen Teambesprechungen & a) gesamtes Team der Station inkl. GB & \\
\hline & b) überregionalen GB-Treffen & b) GB & \\
\hline $\begin{array}{l}\text { Feldkontakt 2: } \\
\text { "Interviews“ }\end{array}$ & qualitative, semistrukturierte Interviews & GB & $\begin{array}{l}\text { Ermittlung subjektiver Erfahrungen und } \\
\text { Einschätzungen von GB bezüglich ihrer } \\
\text { Akzeptanz und Integration ins Team sowie } \\
\text { ihrer Arbeit mit Patienten }\end{array}$ \\
\hline \multirow{4}{*}{$\begin{array}{l}\text { Feldkontakt 3: } \\
\text { „Fokusgruppen“ }\end{array}$} & moderierte Gesprächsgruppen & & \multirow{4}{*}{$\begin{array}{l}\text { Diskussion der Ergebnisse aus den } \\
\text { Feldkontakten } 1 \text { und } 2 \text { mit den Teilneh- } \\
\text { mern, dadurch Validierung und Korrektur; } \\
\text { Entwicklung von Verbesserungs- und } \\
\text { Lösungsansätzen für die Integration der GB }\end{array}$} \\
\hline & a) an den jeweiligen Kliniken & $\begin{array}{l}\text { a) Vertreter verschiedener Berufs- } \\
\text { gruppen (Pflegende, Ärzte, Thera- } \\
\text { peuten, Sozialarbeiter u. a.) }\end{array}$ & \\
\hline & b) im AK „Trialog konkret“ & b) Mitglieder des AK & \\
\hline & $\begin{array}{l}\text { c) im Rahmen eines überregionalen } \\
\text { GB-Treffens }\end{array}$ & c) GB & \\
\hline
\end{tabular}

\section{Zentrale Fragestellungen des Projekts}

- Wie gelingt die Integration von Genesungsbegleitern in psychiatrische Behandlungsteams? Wie erleben die unterschiedlichen Akteure den Integrationsprozess?

- Welche Rollen und Aufgaben übernehmen Genesungsbegleiter?

- Welche Hindernisse treten auf und welche Strategien werden zu ihrer Überwindung entwickelt?

- Welche spezifischen Fähigkeiten besitzen Genesungsbegleiter?

- Welchen Einfluss können sie auf die Anwendung von Zwangsmaßnahmen haben?

Zur Klärung dieser Forschungsfragen verfolgte das hier vorgestellte Projekt ein primär qualitativ-empirisches Forschungsdesign, da sich derart komplexe Interventionen wie die Integration von Genesungsbegleitern und damit zusammenhängende soziale Prozesse am besten mithilfe von Methoden aus der qualitativ-empirischen Sozialforschung untersuchen lassen $[5,6]$. Eine rein quantitativ-empirische Herangehensweise hingegen kann dazu führen, dass patientenrelevante Aspekte der Arbeit von Genesungsbegleitern nicht hinreichend abgebildet werden und der Eindruck entsteht, diese habe keinen nennenswerten Nutzen [7, 8]. Auch Mahlke et al. fordern die Ergänzung quantitativer Designs durch qualitative Ansät- ze und haben letztere bereits in laufende Forschungsprojekte integriert $[9,10]$. In diesem Beitrag stellen wir das primär qualitativ-empirische Forschungsdesign dar, präsentieren ausgewählte Baseline-Daten und diskutieren erste Teilergebnisse der qualitativ-empirischen Interviewstudie im Hinblick auf den Einfluss von Genesungsbegleitern auf die Anwendung und Reduktion von Zwangsmaßnahmen.

\section{Methoden}

Zur Vorbereitung auf die einzelnen Forschungsschritte der vorgestellten Studie (Tab. 1) fand in jeder der beteiligten Kliniken zwischen Dezember 2015 und Januar 2016 eine Mitarbeiterschulung samt Aufklärung über die Studienziele und Arbeitspakete statt. Im Anschluss an die Mitarbeiterschulung wurde eine standardisierte Befragung mittels eigens für die Studie entwickelter Kurzfragebögen durchgeführt, welche einerseits von den jeweiligen Genesungsbegleitern und andererseits von der jeweiligen Pflegedienstleitung ausgefüllt wurden. Diese Kurzfragebögen beinhalteten 10 bzw. 12 Items, welche Auskunft über die wöchentliche Arbeitszeit und Aufgabenbereiche der Genesungsbegleiter, Vorerfahrungen (sowohl der Genesungsbegleiter als auch der Kliniken) sowie über Wünsche und Ziele der Genesungsbegleiter bezogen auf ihre Arbeitsstelle gaben. 
- Tab. 2 Ergebnisse I der Baseline-Erhebung: Anzahl Genesungsbegleiter und Vorerfahrungen an den Projektkliniken

\begin{tabular}{|c|c|c|}
\hline Klinik & Anzahl & Vorerfahrungen \\
\hline Gütersloh & 1 (initial 2) & $\begin{array}{l}1 \text { EX-IN-Praktikant, dieser im Verlauf als GB im } \\
\text { Projekt angestellt }\end{array}$ \\
\hline \multirow[t]{2}{*}{ Herten } & \multirow[t]{2}{*}{2} & $\begin{array}{l}3 \text { EX-IN-Praktikanten ( } 1 \text { auf geschlossener Station, } \\
2 \text { auf offenen Stationen) }\end{array}$ \\
\hline & & $\begin{array}{l}1 \text { bereits angestellter GB, dieser im Verlauf im } \\
\text { Projekt tätig }\end{array}$ \\
\hline Lengerich & 2 & $\begin{array}{l}5 \text { EX-IN-Praktikanten, davon } 2 \text { als GB im Verlauf im } \\
\text { Projekt angestellt }\end{array}$ \\
\hline Marsberg & 1 & 1 EX-IN-Praktikant \\
\hline \multirow[t]{2}{*}{ Münster } & \multirow[t]{2}{*}{2} & $\begin{array}{l}3 \text { EX-IN-Praktikanten, davon } 1 \text { als GB im Verlauf im } \\
\text { Projekt angestellt }\end{array}$ \\
\hline & & $\begin{array}{l}1 \text { bereits angestellter GB, dieser im Verlauf im } \\
\text { Projekt tätig }\end{array}$ \\
\hline
\end{tabular}

Nach Abschluss der initialen Implementierungsphase wurde mit nicht teilnehmenden, offenen Beobachtungen der multiprofessionellen Teambesprechungen in 3 der beteiligten psychiatrischen Kliniken begonnen, die zwischen April und Oktober 2016 durchgeführt wurden. Die ungefähr im Rhythmus von 3 Monaten durchgeführten überregionalen Treffen der Genesungsbegleiter, die insbesondere dem standortübergreifenden offenen Austausch untereinander dienten, wurden ebenfalls in die Beobachtungen eingeschlossen. Insgesamt wurden 25 multiprofessionelle Teambesprechungen und 5 überregionale Genesungsbegleitertreffen beobachtet. In den nächsten methodischen Schritten wurden 9 qualitative, semistrukturierte Interviews mit den Genesungsbegleitern (bei einem Genesungsbegleiter erfolgte im Verlauf ein zweites Interview) sowie eine homogene und 5 heterogene Fokusgruppen durchgeführt. Die 9 Interviews mit den Genesungsbegleitern wurden unter Zuhilfenahme eines im Verlauf regelmäßig angepassten Leitfadens semistrukturiert und per Audioaufzeichnung mitgeschnitten. Alle Genesungsbegleiter hatten im Vorfeld sowohl dem Interview als auch der Audioaufzeichnung zugestimmt. Für die weiteren Analyseschritte wurden die Audiodateien der Interviews wörtlich transkribiert und umfassend anonymisiert. Die Textdateien wurden unter Verwendung der Software MAXQDA Plus 12 induktiv und deduktiv codiert. Diese Analyseschritte erfolgten in Zusammenarbeit mit allen Autoren, sodass sowohl eventuelle Vorannahmen durch verschiedene berufliche Perspektiven (Psychiatrie, Soziologie, Medizinethik, Philosophie, Peer-Arbeit) als auch der eigene fachliche Hintergrund vor Analysebeginn gründlich reflektiert werden konnten. Das Codieren der umfangreichen Daten folgte dem Prinzip der qualitativen Inhaltsanalyse [12]. Alle Ergebnisse wurden in Team- sitzungen diskutiert und die abschließende Interpretation der Daten durch alle Autoren kritisch überprüft.

\section{Ergebnisse}

\section{Baseline-Daten}

Alle 5 Kliniken verfügten über unterschiedliche Vorerfahrungen mit Genesungsbegleitern, insbesondere durch Praktika im Rahmen der EX-IN-Ausbildung ( $>$ Tab. 2). Die wöchentliche Arbeitszeit, die initial in den jeweiligen Kliniken zwischen Pflegedienstleitung und Genesungsbegleitern vereinbart worden war, war heterogen mit einer Spannweite von 5 bis 20 Stunden. Die Genesungsbegleiter arbeiteten auf offenen Stationen (wobei einer im Verlauf auf eine geschlossene Akutstation wechselte), ein Genesungsbegleiter in einer Tagesklinik. Es gab einige Genesungsbegleiter, die in der Vergangenheit bereits selbst Patienten in der Klinik waren, in der sie eingestellt wurden (3 von 8). Die Tätigkeiten sämtlicher Genesungsbegleiter umfassten Einzel- und Gruppengespräche mit Patienten sowie variierend verschiedene Gruppenangebote, u. a. „Kreatives Schreiben“, „Soziales Kompetenztraining“ oder „Genusstraining“. Ein Genesungsbegleiter bot Hausbesuche nach Entlassung der Patienten an ( $>$ Tab. 3 ). Die Bezahlung der Genesungsbegleiter erfolgte in der Entgeltgruppe 3a TVöD-KR und wurde an den Kliniken während des Projektzeitraums zur Hälfte aus Projektmitteln und zur anderen Hälfte aus Eigenmitteln bestritten.

\section{Qualitativ-empirische Interviewstudie}

Diejenigen Genesungsbegleiter, die in einer Tagesklinik oder auf einer offenen, nicht akut aufnehmenden allgemeinpsychiatrischen Station eingesetzt waren, hatten im Rahmen ihrer Tätigkeit wenige Berührungspunkte mit Zwangsmaßnahmen. Andere hingegen, die im Rahmen ihrer aktuellen Anstellung oder eines vorherigen Praktikums auf einer Akutstation tätig waren, hatten Erfahrungen mit der Anwendung von Zwangsmaßnahmen gemacht. Hinzu kommt, dass einige Genesungsbegleiter in der Vergangenheit als Patienten selbst Zwang erlebt hatten.

Die befragten Genesungsbegleiter (GB) berichteten, dass ein wichtiges Mittel, um deeskalierend wirken zu können und so den Einsatz von Zwangsmaßnahmen eventuell bereits im Vorfeld verhindern oder verringern zu können, ihr durch ihre eigene Krisenerfahrung besonders geprägter Kontakt zu den Patienten sei:

- GB 1: [...] wenn die halt hören, dass man selber mal solche Krisen hatte und, ja, das könnte ich mir schon vorstellen, dass da der Kontakt vielleicht dann eher entstehen könnte.

- GB 2: Und dadurch, dass ich sagen konnte: ,Ich war selber mal in der Situation. Ich weiß gerade wie es dir geht', wurde er dann wirklich ruhiger. 
- Tab. 3 Ergebnisse II der Baseline-Erhebung: Einsatzort und Tätigkeiten der Genesungsbegleiter

\begin{tabular}{|c|c|}
\hline Einsatzort & Tätigkeiten (unter anderem) \\
\hline Allgemeinpsychiatrische Tagesklinik & Einzel- und Gruppengespräche, Gruppe „Kreatives Schreiben“ \\
\hline Offene allgemeinpsychiatrische Station & $\begin{array}{l}\text { Einzelgespräche, Arbeit mit Tools (Timeline, Genesungs- und Krisenplan), } \\
\text { Begleitung sozialer Aktivitäten }\end{array}$ \\
\hline Offene Station, Schwerpunkt Depression und Persönlichkeitsstörung & Einzelgespräche, Genusstraining, Schreibwerkstatt, Entlassgruppe \\
\hline Offene Station, gemischte Diagnosen & $\begin{array}{l}\text { Einzel- und Gruppengespräche, Begleitung von Patienten, Nachsorge inkl. } \\
\text { Entlassgruppe, Hausbesuche }\end{array}$ \\
\hline $\begin{array}{l}\text { Offene Station mit Schwerpunkt Ersterkrankungen, junge Erwachsene, } \\
\text { Psychosen }\end{array}$ & Einzelgespräche, Soziales Kompetenztraining, Recovery-Gruppe \\
\hline Offene allgemeinpsychiatrische Station & $\begin{array}{l}\text { Patientengespräche, Psychoedukationsgruppe, Recovery- / Salutogene- } \\
\text { se-orientierte Gruppe }\end{array}$ \\
\hline Offene allgemeinpsychiatrische Station & Patientengespräche, Recoverygruppe \\
\hline Ambulanz sowie offene Akutstation, Einsatz als „Springer“ & $\begin{array}{l}\text { Patientengespräche, Recoverygruppe, Begleitung der Patienten außerhalb } \\
\text { der Klinik, Soziales Kompetenztraining, Seniorengruppe }\end{array}$ \\
\hline
\end{tabular}

Des Weiteren wurde die den Genesungsbegleitern in der Regel mehr und insbesondere flexibler zur Verfügung stehende Zeit als Aspekt identifiziert, der bei der Verhinderung von Zwangsmaßnahmen im Vorfeld zum Tragen kommen könnte:

- GB 3: Ich glaube, wenn da jemand so viel Zeit wie ich hätte, eine Stunde mit jemanden zu reden, wenn ich mich eine Stunde einfach zu jemanden dazu setzen könnte, der halt so von der Rolle ist, aufgeregt oder so - einfach nur da sein. Ich könnte mir vorstellen, dass man dadurch eine Fixierung verändern, verhindern könnte.

Ob die eigene Erfahrung mit Zwang für einen Genesungsbegleiter hilfreich sein kann, um Zwang bei Patienten entgegenzuwirken, wurde von den Interviewpartnern intensiv reflektiert. Dabei stand erneut der als „besonders“ wahrgenommene Kontakt zu den Patienten im Mittelpunkt, der vor allem über die ähnlichen Krisenerfahrungen zustande komme. Die eigenen Krisenerfahrungen mit zum Teil eigenem Erleben von Zwangsmaßnahmen wurden von den Genesungsbegleitern aber auch als problematisch wahrgenommen, insbesondere weil sie bei der Anwendung von Zwangsmaßnahmen bei Patienten eine mögliche „Triggerquelle“ darstellen können:

- GB 1: [...] nicht dass dann nachher wieder was hochkommt und die [die Genesungsbegleiter, Anm. der Autoren] sich dann daneben legen können, sage ich jetzt mal ganz krass, ich denke mal, da muss man sich gut kennen und auch auf sich aufpassen [...].

- GB 7: Ich denke das ist gut für den Patienten, weil ich das nachvollziehen kann. Ich muss nur wegen mir aufpassen, dass das mich nicht so sehr runter zieht.

Als zusätzlichen Gesichtspunkt führte ein Interviewpartner an, dass man als Genesungsbegleiter nicht nur auf die Patienten selbst, sondern auch auf die anderen Teammit- glieder einwirken und so zu einer Deeskalation beitragen könne:

- GB 4: Dass Genesungsbegleiter, die selber auch Erfahrung haben damit, dass sie auch dem Kollegen gegenüber benennen können, gegenüber welchen Dingen sie auch im Vorfeld aufmerksamer sind. Im Vorfeld, auf das und das da müsst ihr aufpassen. Damit es gar nicht eskaliert.

\section{Diskussion}

\section{Studiendesign und Baseline-Daten}

Das hier vorgestellte Projekt befasste sich mit der Integration von Genesungsbegleitern in psychiatrischen Krankenhäusern, die bis dahin geringe Vorerfahrungen mit der Arbeit von Genesungsbegleitern hatten. Die Initiierung und finanzielle Förderung durch den Träger (LWL) und die im Projektverlauf enge Anbindung und Rückkopplung an den trialogisch besetzten AK „Trialog konkret“ leisteten dabei einen wichtigen Beitrag zum gegenseitigen Austausch und zur vernetzten Entwicklung der Arbeit von Genesungsbegleitern im LWL-PsychiatrieVerbund. Die parallele Förderung und Umsetzung eines wissenschaftlichen Evaluationsprojekts (an dem im Sinne einer Peer-Partizipation in der Forschung mit A.W. eine Genesungsbegleiterin beteiligt war) stellten im Hinblick auf eine anvisierte dauerhafte Etablierung von Genesungsbegleitern in psychiatrischen Behandlungsteams eine wichtige Ergänzung dar, da die Ergebnisse der Forschung dazu beitragen können, dass Hindernisse und Herausforderungen der Integration identifiziert und erfolgreiche Lösungsansätze entwickelt werden können.

Mit Blick auf die Ergebnisse der Baseline-Erhebung zeigt sich, dass die Umsetzung der Arbeit von Genesungsbegleitern über die verschiedenen Kliniken hinweg heterogen ausfiel. Diese Heterogenität war für die eingangs genannten Forschungsfragen jedoch nicht von Nachteil, sondern 
stellte sich im Gegenteil bei der Untersuchung des Integrationsprozesses von Genesungsbegleitern und ihrer Rollenfindung als wertvoll heraus. Die regelmäßig, an wechselnden Standorten durchgeführten überregionalen Genesungsbegleitertreffen, gegen deren Ende jeweils ein Mitglied des AK „Trialog konkret“ hinzukam, boten Genesungsbegleitern dabei die Möglichkeit, sich gegenseitig auszutauschen und etwaige Wünsche und Probleme an den AK weiter zu tragen. Dadurch konnten die Genesungsbegleiter nicht nur voneinander lernen und in ihrer jeweiligen Rolle bestärkt und gefestigt werden, sondern auch konkrete Unterstützung durch den beim Träger angesiedelten AK bekommen, z. B. durch die Finanzierung eines Supervisors. Der AK „Trialog konkret“ blieb somit in seiner Funktion der Entwicklung und Begleitung des Projekts unmittelbar an die Genesungsbegleiter angebunden.

Bezüglich des gewählten primär qualitativ-empirischen Forschungsansatzes kann konstatiert werden, dass sich ein derartiges methodisches Vorgehen vor dem Hintergrund der Offenheit des Implementierungsprozesses und der limitierten Vorerfahrungen mit Genesungsbegleitern in den jeweiligen Kliniken als hilfreich und umsetzbar erwiesen hat. Die Einnahme einer solchen explorativen und offenen Haltung im Forschungssetting erscheint besonders sinnvoll, um die soziale Realität an den jeweiligen Kliniken möglichst breit gefächert erfassen und abbilden zu können.

\section{Einfluss der Genesungsbegleiter auf die Anwendung von Zwang}

In einer von Steinert et al. durchgeführten Online-Befragung von ärztlichen Leitern psychiatrischer Kliniken in Deutschland hatten knapp 24\% angegeben, dass der Einsatz von Genesungsbegleitern auf psychiatrischen Stationen dazu beitragen könne, freiheitseinschränkende Maßnahmen in der Psychiatrie zu reduzieren [13]. Die von uns interviewten Genesungsbegleiter sahen in ihrer Arbeit ebenfalls das Potenzial, der Anwendung von Zwang vorzubeugen bzw. Zwang in der Psychiatrie zu reduzieren. Dabei identifizierten sie zum einen unspezifische Faktoren wie das ihnen in aller Regel flexibler zur Verfügung stehende Zeitkontingent, wodurch mehr Ressourcen für deeskalierende Maßnahmen im Einzelfall vorhanden seien. Auf der anderen Seite wiesen die Interviewpartner auch auf spezifische Fähigkeiten ihrer Berufsgruppe hin. Durch ihre oftmals eigene Krisenerfahrung könnten Genesungsbegleiter einen authentischen und in gewisser Hinsicht „besonderen“ Zugang zu Patienten in psychischen Ausnahmesituationen herstellen und deeskalierend und präventiv tätig werden, bevor es zu einer Zwangsmaßnahme kommt. Auf der Suche nach Alternativen zu freiheitsentziehenden Maßnahmen wäre der Einsatz von Genesungsbegleitern auf psychiatrischen Akutstationen in diesem Sinne ein innovativer Ansatz, der aus diesem Grund weiter erprobt und wissenschaftlich evaluiert werden sollte. Dabei sollte in jedem Fall darauf geachtet werden, dass Genesungsbe- gleiter nur dann im Akutbereich eingesetzt werden, wenn sie sich dies auch explizit zutrauen, und dass durch geeignete Maßnahmen - zum Beispiel durch regelmäßige Nachbesprechungen oder Supervisionen - dafür Sorge getragen wird, dass Genesungsbegleiter durch das Miterleben von Zwangsmaßnahmen nicht selbst psychisch destabilisiert werden.

\section{Stärken und Schwächen der Studie}

Aus dem angelsächsischen Bereich sind qualitativ-empirische Arbeiten zu Genesungsbegleitern bekannt, die 2013 von Walker und Bryant in einer Metasynthese zusammengefasst worden sind [11]. Nach unserem Kenntnisstand ist unsere Studie eine der ersten Untersuchungen, die die Integration von Genesungsbegleitern unter Einsatz von 3 qualitativ-empirischen Methoden im deutschsprachigen Raum untersucht.

Als besondere Stärke unserer Studie ist somit der Einsatz verschiedener qualitativer Methoden (Beobachtungen, Interviews und Fokusgruppen) hervorzuheben, welche es den Beteiligten ermöglicht haben, ihre Erfahrungen und Eindrücke bezüglich der Integration von Genesungsbegleitern in akutpsychiatrische Behandlungsteams ausführlich zu schildern. Das so gewonnene Datenmaterial erlaubt ein umfassenderes und stärker kontextualisiertes Verständnis der Situation aus Sicht der Akteure als beispielsweise durch rein quantitative Ansätze gewonnene Daten. Auch das durch die qualitative Methodenwahl bewusst flexibel gestaltete Design der Studie hat sich in der Praxis als wertvoll erwiesen, da so auf kurzfristige Veränderungen (Wechsel von Stationen, kurzfristiges Ausscheiden, kurzfristige oder zeitlich verzögerte Neueinstellungen), die jede Pilotimplementierung mit sich bringen kann, rechtzeitig reagiert und so auch wichtige Daten über diese veränderten Situationsbedingungen gewonnen werden konnten.

Limitierend ist anzumerken, dass die Kontextualisierung qualitativer Daten aus subjektgebundener Perspektive geschieht und dementsprechend eine Generalisierung der Daten, wie beispielsweise bei quantitativen Designs, nicht ohne weiteres zulässig ist. Die Wahl der Interviewmethode birgt das Risiko, dass Interviewpartner, insbesondere bei sensibleren Themen, kontrovers eingeschätzte Meinungen zugunsten von sozial erwünschteren Aussagen aufgeben. Die Zusicherung umfassender Anonymisierung, der Aufbau eines Vertrauensverhältnisses zum Forscher sowie die in den Daten abgebildete Diversität an Aussagen und Meinungen führen uns jedoch zu dem Schluss, dass eine mögliche Verzerrung unserer Daten durch soziale Erwünschtheit eher gering ausgefallen ist. Um mit der Methode einhergehende Verzerrungs- und Fehlerquellen dennoch auf ein Minimum zu reduzieren, die Subjektivität der Daten zu mindern und diese auf Plausibilität zu überprüfen, wurden die Beobachtungs- und Interviewdaten der Teilnehmer in 6 Fokusgruppen mit den unterschiedlichen Akteuren diskutiert, ergänzt und so methodisch trianguliert. 


\section{FAZIT}

Die Integration von Genesungsbegleitern in psychiatrische Behandlungsteams wirft verschiedene Fragen auf, u. a. welche konkreten Aufgaben durch Genesungsbegleiter übernommen werden sollen. Die Unterstützung durch den Klinikträger und Ansprechpartner in den jeweiligen Kliniken sowie überregionale Treffen inkl. Supervisionsangebot ermöglichen Genesungsbegleitern, sich auszutauschen und so ihre Rollen und Aufgabenfelder zu finden.

Der Einsatz von Genesungsbegleitern kann einen spezifischen Beitrag dazu leisten, durch deeskalierende Maßnahmen die Anwendung von Zwang in der Psychiatrie zu reduzieren.

\section{Interessenkonflikte}

Es liegen keine Interessenkonflikte vor.

\section{Danksagung}

Dem Landschaftsverband Westfalen-Lippe (LWL) und der LWL-Abteilung für Krankenhäuser und Gesundheitswesen, LWL-PsychiatrieVerbund Westfalen (Prof. Dr. M. Noeker; T. Profazi) danken wir für die finanzielle und ideelle Förderung des Projekts (Projektlaufzeit 09/2015-09/2017). Ferner danken wir allen Mitgliedern des AK „Trialog konkret“ des LWL für ihr Engagement und ihre konstruktive Begleitung des Projekts. Prof. Dr. Jan Schildmann danken wir für seine methodische Beratung. Schließlich danken wir allen Genesungsbegleitern und Mitarbeitern der beteiligten LWL-Kliniken für ihre Mitarbeit und Unterstützung unserer Forschung.

\section{Korrespondenzadresse}

\section{Dr. med. Jakov Gather, M.A.}

LWL-Forschungsinstitut für Seelische Gesundheit LWL-Universitätsklinikum, Ruhr-Universität Bochum Alexandrinenstr. 1-3, 44791 Bochum Tel. 0234/50774416, Fax 0234/507744339 jakov.gather@rub.de

\section{Literatur}

[1] Davidson L, Bellamy C, Guy K, Miller R. Peer support among persons with severe mental illnesses: a review of evidence and experience. World Psychiatry 2012; 11(2): 123-128.

[2] Mahlke C, Krämer U, Kilian R, Becker T. Bedeutung und Wirksamkeit von Peer-Arbeit in der psychiatrischen Versorgung: Übersicht des internationalen Forschungsstandes. Nervenheilkunde 2015; 34(4): 235-239.

[3] Lange K. Erfahrene verändern die Psychiatrie - Europäisches Pilotprojekt EX-IN schafft Modelle der Nutzerbeteiligung. Psychosoziale Umschau 2008; 23(1): 4-5.

[4] Utschakowski J, Sielaff G, Schulz G. Aus Erfahrung wird Wissen und Kompetenz: Ausbildung von Experten durch Erfahrung. Nervenheilkunde 2015; 34(4): 271-274.

[5] Petermann F. Implementationsforschung: Grundbegriffe und Konzepte. Psychologische Rundschau 2014; 65: 122-128.

[6] Wäscher S, Salloch S, Ritter P, Vollmann J, Schildmann J. Triangulation in der empirischen Medizinethik am Beispiel des ETHICO-Projektes („Empirical-Ethical Interventions in Oncology“). Bioethica Forum 2014; 7(4): 121-130.

[7] Hardiman ER. No difference in psychosocial and mental health outcomes for clients receiving statutory mental health services from consumer-providers compared to professionals. Evidence-Based Mental Health 2013; 16(3): 80.

[8] Pitt V, Lowe D, Hill S, Prictor M, Hetrick SE, Ryan R, Berends L. Consumer-providers of care for adult clients of statutory mental health services. The Cochrane database of systematic reviews 2013; 3: CD004807.

[9] Mahlke C, Krämer U, Becker T, Bock T. Peer support in mental health services. Current Opinion in Psychiatry 2014; 27(4):276-281.

[10] Mahlke C, Heumann K, Ruppelt F, Meyer H-J, Rouhiainen T, Sielaff G, Lambert M, Bock T. Peer-Begleitung für Erfahrene schwerer und langfristiger psychischer Störungen und Angehörige - Hintergrund, Konzept und Baseline-Daten. Psychiatrische Praxis 2015; 42 Suppl 1: S25-29.

[11] Walker G, Bryant W. Peer support in adult mental health services: A metasynthesis of qualitative findings. Psychiatric Rehabilitation Journal 2013; 36(1): 28-34.

[12] Mayring P. Qualitative Inhaltsanalyse. Grundlagen und Techniken. Basel, Weinheim: Beltz 2003.

[13] Steinert T, Schmid P, Arbeitskreis zur Prävention von Gewalt und Zwang, Landesverband der Psychiatrie-Erfahrenen Baden-Württemberg. Zwangsmaßnahmen in psychiatrischen Kliniken in Deutschland. Gegenwärtige Praxis (2012). Nervenarzt 2014; 85: 621-629.

Bibliografie

DOI https://doi.org/10.1055/a-0829-0436

Nervenheilkunde 2019; 38: 184-189

(c) Georg Thieme Verlag KG Stuttgart · New York ISSN 0722-1541 
Punkte sammeln auf CME.thiemede

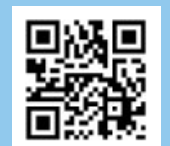

Diese Fortbildungseinheit ist in der Regel 12 Monate online für die Teilnahme verfügbar. Den genauen Einsendeschluss finden Sie unter https://eref.thieme.de/CXCGYPG.

Sollten Sie Fragen zur Online-Teilnahme haben, finden Sie unter https://cme.thieme.de/hilfe eine ausführliche Anleitung. Wir wünschen viel Erfolg beim Beantworten der Fragen!

Unter https://eref.thieme.de/CXCGYPG oder über den QR-Code kommen Sie direkt zur Startseite des Wissenstests.

VNR 2760512019156645333

\section{Frage 1}

Was ist ein Genesungsbegleiter?

A Ein Mensch in einer akuten psychischen Krise, der in seiner Genesung begleitet wird.

B Ein speziell auf dem Gebiet der psychischen Erkrankungen geschulter Sozialarbeiter.

C Ein selbst von einer psychischen Erkrankung Betroffener, der nach einer spezifischen Ausbildung seine Erfahrung und seine Kompetenzen in die Behandlung von Menschen in psychischen Krisen einbringt.

D Ein ehrenamtlicher Helfer, der somatisch Kranken bei der Wiedergesundung hilft.

E Ein Studierender des sozialen oder medizinischen Fachbereichs, der im Rahmen einer Nebentätigkeit an der Behandlung psychisch erkrankter Menschen beteiligt wird.

\section{Frage 2}

Welche der folgenden Aussagen trifft im Hinblick auf die Arbeit von Genesungsbegleitern im psychiatrischen Versorgungssystem zu?

A In Deutschland wird die Ausbildung zum Genesungsbegleiter an einem zentralen Schulungsort (Berlin) angeboten.

B In angelsächsischen Ländern ist die Arbeit von Genesungsbegleitern in der Regel deutlich weniger etabliert als in Deutschland.

C Seit den frühen 1960er Jahren gibt es ein starkes wissenschaftliches und klinisches Interesse an der Arbeit von Genesungsbegleitern.

D In Deutschland ist jedes psychiatrische Krankenhaus gesetzlich verpflichtet, mindestens einen Genesungsbegleiter zu beschäftigen.

E Genesungsbegleiter suchen zunehmend nach Anstellungsmöglichkeiten im psychiatrischen Versorgungssystem.

\section{Frage 3}

Wie ist das Ausbildungscurriculum zum Genesungsbegleiter in Deutschland organisiert?

A Als Bachelorstudium an einer privaten Universität.

B Als mehrjährige, berufsbegleitende Zusatzqualifikation.

C Als einjährige Ausbildung nach dem „EX-IN“-Curriculum.

D Als drei dreißigtägige Praktika, die durch eine mündliche Prüfung abgeschlossen werden.

E Als einjährige Ausbildung ohne festgelegten Lehrplan.

\section{Frage 4}

Welche Aussage zu qualitativ-empirischen Forschungsmethoden trifft zu?

A Qualitativ-empirische Methoden ermöglichen in der Regel ein weniger stark kontextualisiertes Verständnis der Sicht der Akteure als quantitativ-empirische Methoden.

B Unterschiedliche qualitativ-empirische Methoden sind miteinander kombinierbar, um die Erfahrungen und Einstellungen der Beteiligten umfassend abzubilden.

C Qualitativ-empirische Methoden sind quantitativ-empirischen Methoden in jeder Hinsicht unterlegen und sollten generell als zweite Wahl angesehen werden.

D Qualitativ-empirische Methoden sind stets weniger ressourcenaufwändig als quantitativ-empirische Methoden.

E Im angelsächsischen Bereich liegen so gut wie keine Studien mit qualitativ-empirischen Methoden zum Thema „Genesungsbegleiter“ vor.

\section{Frage 5}

Welche der folgenden Aufzählungen enthält ausschließlich qualitativ-empirische Methoden?

A Beobachtung, strukturierte Umfrage, Experiment

B Fokusgruppe, Beobachtung, Interview

C Interview, Fokusgruppe, Fragebogen

D Fragebogen, Beobachtung, strukturierte Umfrage

E Strukturierte Umfrage, Fokusgruppe, Beobachtung

\section{Frage 6}

Welche Aussage zu den Baseline-Daten der Studie ist zutreffend?

A Die wöchentliche Arbeitszeit der Genesungsbegleiter war heterogen und wurde individuell zwischen Genesungsbegleitern und Pflegedienstleitung vereinbart.

B Es fand sich eine Mindestarbeitszeit von 10 Stunden pro Woche.

C Genesungsbegleiter arbeiteten ausschließlich auf geschlossenen Stationen.

D Baseline-Daten wurden in der Mitte der Projektlaufzeit erhoben und laufend aktualisiert.

E Die Baseline-Daten wurden durch Beobachtungen erhoben.

- Weitere Fragen auf der folgenden Seite ... 


\section{Punkte sammeln auf CME.thieme.de}

Fortsetzung $\ldots$

\section{Frage 7}

Welche Aussage zu überregionalen Genesungsbegleitertreffen ist korrekt?

A Bei überregionalen Genesungsbegleitertreffen können sich Genesungsbegleiter unterschiedlicher Standorte untereinander austauschen und gegenseitig von ihren Erfahrungen und Problemen lernen.

B Unter überregionalen Genesungsbegleitertreffen versteht man in der Regel jährlich stattfindende Treffen von Genesungsbegeitern aus unterschiedlichen Ländern.

C Überregionale Genesungsbegleitertreffen können nicht mit einer Supervision verknüpft werden.

D Überregionale Genesungsbegleitertreffen sollten ärztlich geleitet werden.

E Überregionale Genesungsbegleitertreffen machen nur Sinn, wenn sie mindestens 1 Mal pro Monat stattfinden.

\section{Frage 8}

Welche der folgenden Aussagen zum Einfluss von Genesungsbegleitern auf die Anwendung von Zwang in der Psychiatrie ist falsch?

A Es wird darüber diskutiert, ob bzw. inwiefern Genesungsbegleiter dazu beitragen können, die Anwendung von Zwang in der Psychiatrie zu reduzieren.

B Genesungsbegleiter können aufgrund ihrer eigenen Krisenerfahrung unter Umständen einen besonderen Zugang zu Patienten aufbauen und dadurch deeskalierend wirken.

C Die Teilnahme von Genesungsbegleitern bei der Anwendung von Zwang birgt die Gefahr, dass die Genesungsbegleiter selbst psychisch belastet werden, insbesondere wenn sie in der Vergangenheit selbst Zwangsmaßnahmen erleben mussten.

D Zu dem Thema liegen bereits viele empirische Daten vor, da viele Genesungsbegleiter auf Akutstationen arbeiten und in der Vergangenheit in Studien eingeschlossen worden sind.

E Die Zeit, die den Genesungsbegleitern häufig in höherem und flexiblerem Maße zur Verfügung steht, kann unter Umständen zu einer Deeskalation und Beruhigung einer angespannten Situation beitragen.

\section{Frage 9}

Welche Aussage zu den Stärken und Schwächen der Studie trifft zu?

A Qualitativ-empirische Studien erlauben in der Regel besser generalisierbare Aussagen als quantitativ-empirische Studien.

B Methodisch bedingte Verzerrungs- und Fehlerquellen können reduziert werden, indem eine methodische Triangulation vorgenommen wird.

C Interviewteilnehmer antworten in der Regel objektiv.

D Das Studiendesign ließ keine Anpassung an kurzfristige Veränderungen (z. B. Wechsel von Genesungsbegleitern auf andere Stationen) zu.

E Die Verzerrung der Daten durch soziale Erwünschtheit ist auf jeden Fall als hoch anzusehen.

\section{Frage 10}

Welche der folgenden Aufgaben wurde während der Projektlaufzeit nicht von Genesungsbegleitern übernommen?
A Einzelgespräche
B Recoverygruppe
C Begleitung sozialer Aktivitäten
D Soziales Kompetenztraining
E Medikamentenberatung 